\title{
Clinical Profile and the Management of Mycotic Superior Mesenteric Artery (SMA) Aneurysm in Infective Endocarditis: A Potential Tsunami
}

\author{
Prakash Sanzgiri ${ }^{1}$, Charan Reddy Kudumula ${ }^{1^{*}}$, Kaushal Pandey ${ }^{2}$, Dattaprassana R Kulkarni ${ }^{3}$ \\ ${ }^{l}$ Department of Clinical and Interventional Cardiology \\ ${ }^{2}$ Department of Cardiovascular and Thoracic Surgery \\ ${ }^{3}$ Department of Gastrointestinal Surgery, Lilavati Hospital and Research Centre, Mumbai, India
}

\begin{abstract}
*Corresponding Author: Charan Reddy Kudumula, Resident doctor Cardiology A Department, IbnSina Teaching Hospital Rabat, E-mail: chrnr@ rediffmail.com
\end{abstract}

\begin{abstract}
Visceral artery aneurysms are uncommon, often found incidentally and carry significant mortality if they rupture. Aneurysms of superior mesenteric artery (SMA) represent only a small minority of the splanchnic aneurysms, but can present with a wide range of symptoms. Mycotic or infective etiology account for more than 50\% of these aneurysms. Diagnosis and early intervention remains the mainstay in its management before any complications. The aim of this report is to highlight the emergent nature of the aneurysms of SMA disease and multidisciplinary approach needed to reduce overall morbidity and mortality. Here, we discuss a rare case of mycotic aneurysms of SMA in infective endocarditis.
\end{abstract}

Keywords: Mycotic aneurysm, Infective endocarditis, Coronary angiography

\section{INTRODUCTION}

William Osler first described mycotic aneurysms in the setting of endocarditis in the year 1885. Superior mesenteric artery (SMA) aneurysms accounts for only $3.2 \%$ of all reported cases [1]. With increase in incidence of intravenous drug abuse, immune-suppression in immune deficiency virus (HIV) infection and cancer chemotherapy and various intravascular interventions, the cases of mycotic aneurysms (MA) are now steadily increasing. Currently, there is no consensus on their diagnosis, treatment and follow-up. Treatment of infective endocarditis coupled with various techniques such as aneurysm resection with or without bowel sparing, saphenous vein graft repair and endovascular stenting are some of the methods used to treat SMA aneurysms secondary to valve endocarditis.

\section{CASe Report}

A 64-year old, non-diabetic, non-hypertensive male presented to us with the history of lowgrade fever and weight loss with anorexia for last three months, and abdominal pain for last one month. He was treated at a local hospital with various oral antibiotics without ascertaining the cause, leading to no improvement in his symptoms. Past history revealed no tuberculosis, cardiovascular disease (CVD), intravenous drug abuse, prolonged hospitalization or any vascular interventions.

On examination, vitals were stable. Jugular venous pulsation (JVP) was normal. On palpation of abdomen, mild tenderness in periumbilical region without any organomegaly was observed. On auscultation of chest, a loud P2 in the second left intercostals space with a grade III pansystolic murmur at the apex radiating to back. No clinical evidence of cardiac failure (S3 gallop or S4) was noted.

Routine blood analysis showed elevated leucocyte count $(22,200 / \mathrm{cu} \mathrm{mm})$. Viral serology was negative. X-ray of chest and abdominal ultrasound examination was also normal. 2Dechocardiography and transesophageal echocardiography (TEE) showed large vegetation $(2.5 \times 1.5 \mathrm{~cm})$ on anterior mitral valve leaflet and small, multiple vegetations on non-coronary cusp of aortic valve (Fig 1). There was moderate mitral and aortic regurgitation. Tricuspid and pulmonary valves were normal. A mild pulmonary hypertension (ePASP $=35 \mathrm{~mm}$ $\mathrm{Hg}$ ) was seen. LV systolic function was normal. No pericardial thickening or effusion observed. 

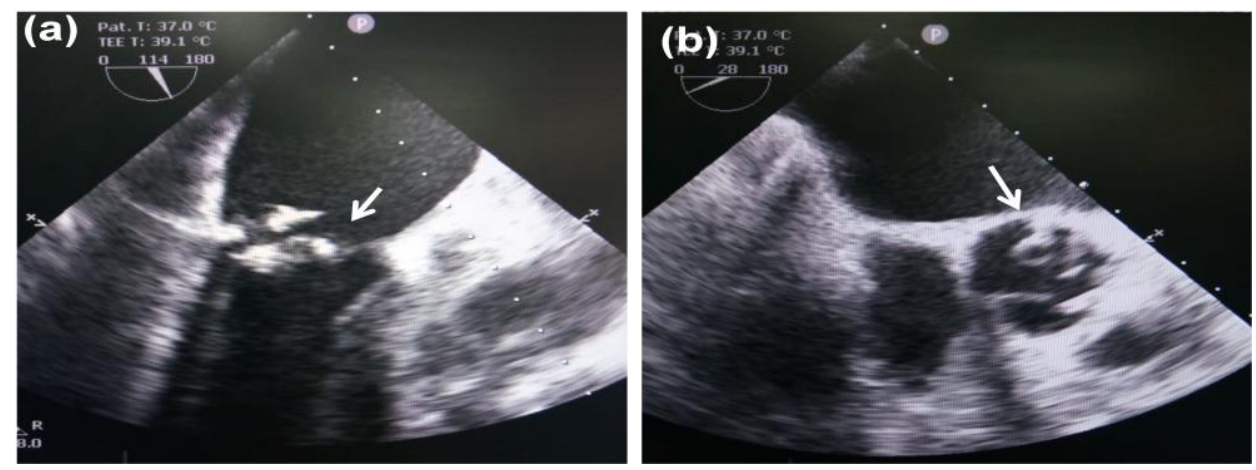

Fig1. Transesophageal echocardiography (TEE) of mitral valve (a) showing large vegetation $(2.5 \times 1.5 \mathrm{~cm})$ on mitral valve leaflet (arrow). TEE of aortic valve (b) showing small multiple vegetations with fenestrations on the non coronary cusp (arrow).

Blood cultures revealed no bacterial growth. Intravenous antibiotic therapy with Ampicillin / Sulbactum (12 gms/day in divided doses) and Gentamicin ( $3 \mathrm{mg} / \mathrm{kg} /$ day) was commenced on admission. Patient was stable but with persistent abdominal discomfort.

PET-CT scan was done, which showed increased FDG uptake on mitral valve leaflet, but no activity in the region of aortic valve (Fig 2A-C). There was also increased FDG activity at the location of two SMA aneurysms. Computerized tomography of abdomen showed
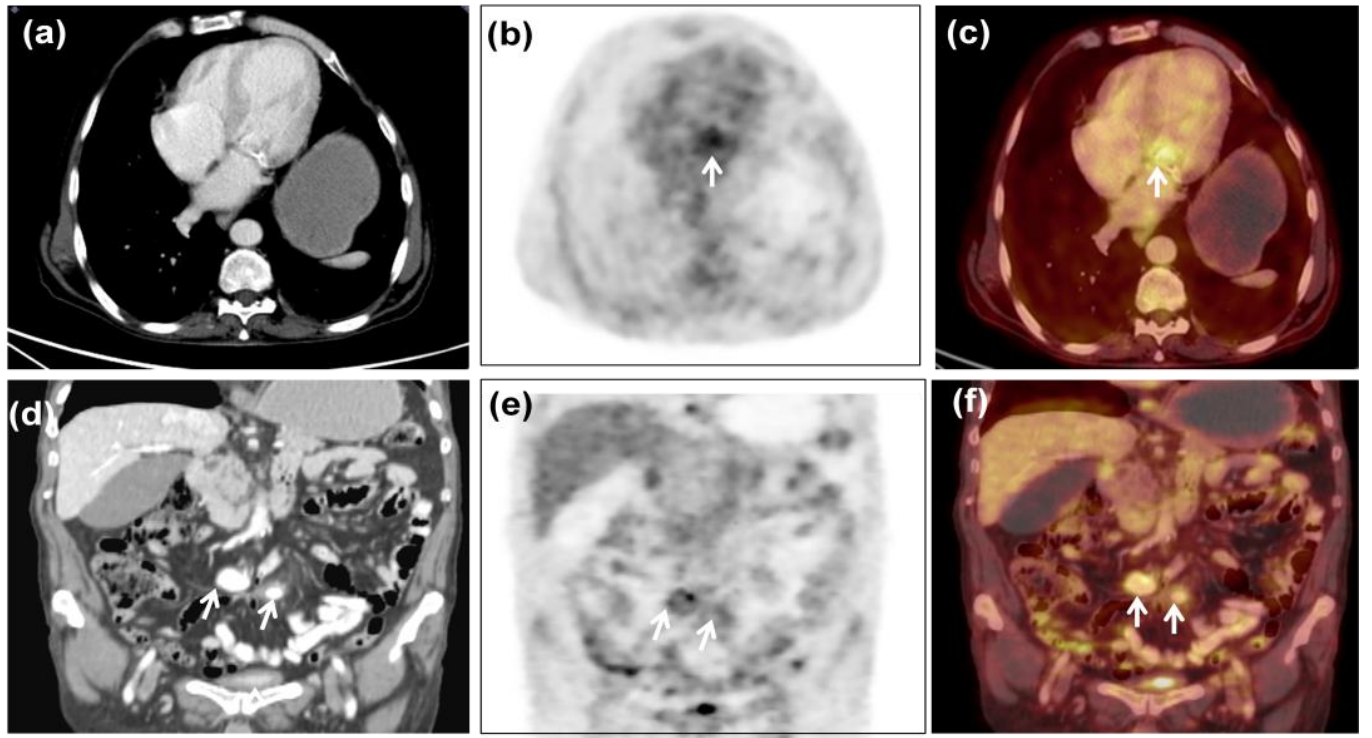

Fig2. Upper panel: Computed Tomography (CT) (a) and Positron Emission Tomography-Computed Tomography $(P E T-C T)$ scan images of chest $(b, c)$ showing high accumulation of FDG isotope at the mitral valve denoting increased metabolic activity (arrow). Lower panel: CT $(d)$ and PET-CT scan images $(e, f)$ of abdomen showing two large aneurysms with increased FDG isotope activity (arrows).

Patient underwent mitral and aortic valve replacement with mechanical prosthetic valves. After three days, patient underwent open abdominal surgery and two SMAA were located and resected along with a segment of ischemic bowel supplied by these arteries with final endto-end anastomosis. Peritoneal fluid was collected for culture and antibiotic sensitivity test. The cultures did not show any bacterial growth. Recovery from both surgeries was uneventful. He was later discharged on warfarin (5mg) and advised to continue antibiotics for 6 weeks.

Gross examination of mitral valve sections revealed focal areas of fibrosis, ulceration in the 
Clinical Profile and the Management of Mycotic Superior Mesenteric Artery (SMA) Aneurysm in Infective Endocarditis: A Potential Tsunami

vicinity of the vegetation on the anterior mitral leaflet (AML) (Fig 3A) with rupture of chodae (Fig 3B,C). Aortic valve showed focal areas of myxoid degeneration with fenestrations of noncoronary cusp and small pale vegetations. Histology of vegetation showed dense neutrophilic and gaint cell infiltration admixed with areas of necrosis and fibrosis. Gram stain, Periodic acid-Schiff (PAS) and Grocott methenamine silver (GMS) (fungal) stains were negative for both the valves
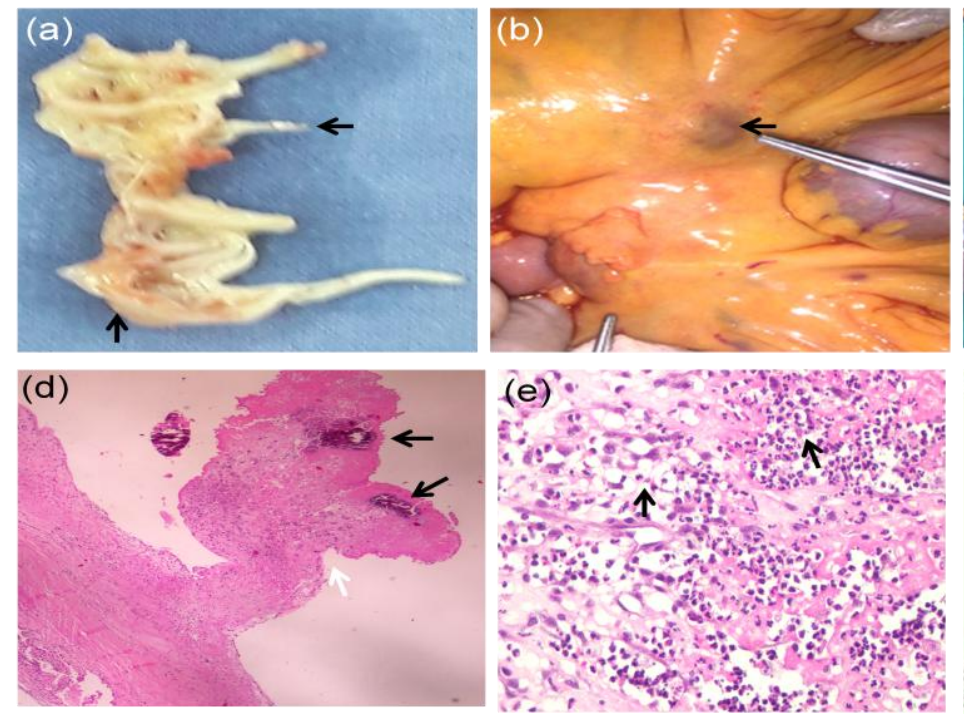

Gross examination of superior mesenteric aneurysms showed two pseudo-aneurysms with few enlarged mesenteric lymph nodes. Gross examination of small bowel (ileum) was pale and oedematous (Fig 3D). Histology of bowel showed few neutrophilic micro-abscesses with areas of necrosis extending into the surrounding adipose tissue (Fig 3E) and early ischemic changes predominantly involving villi, but in few places, full thickness of mucosa and part of sub-mucosa were involved with focal area of stricture, scarring and necrosis (Fig 3F).

Fig3. Upper panel: Surgical specimens showing mitral valve with vegetation (a) (arrow) and chordal rupture (b) pseudoaneuryms of SMA along with resected segment of bowel(c) (arrow).

Lower panel: Histopathological sections of mitral valve vegetation showing focal area of calcification (d) (arrows), mitral vegetation showing dense neutrophilic infiltration with few scattered lymphocytes admixed with histiocytes (e) (40 x magnification of Figure 3d), small bowel showing areas of mucosal ischaemia (f) (arrows) with viable muscularis propria (blue arrow).

\section{DISCUSSION}

The clinical profile of infective endocarditis (IE) is now changing. In pre-antibiotic and early antibiotic era, it typically affected young or middle-aged adults with underlying rheumatic or congenital heart disease (CHD). With the development of antibiotics and its indiscriminate use, and decline in incidence of RHD, lead to a change in the risk factor profile, patient demographic characteristics and microbiology of IE. Double valve endocarditis is uncommon, especially in patient without the history of RHD, vascular cannulation, intravenous (I.V) drug abuse or immune-compromised state. It has been attributed to regurgitant jets from the infected aortic valve striking the anterior mitral leaflet or due to contiguous spread of infection along the aortomitral curtain [2].

Staphylococcus, Streptococcus, Salmonella, atypical Mycobacteria, Coxiella and fungi are usually the infective organisms [3, 4]. Blood culture-negative IE can also be severe and difficult to diagnose. Blood culture-negative IE is classified into three main groups: (a) Bacterial endocarditis sterilized by previous antibiotics therapy. (b) Endocarditis caused by fastidious organisms (e.g. Brucella and Legionella) and cell-dependent organisms (e.g. Chlamydia and Coxiella or fungi), in which prolonged incubation or different techniques are necessary. (c) True blood culture-negative endocarditis, due to intra-cellular bacteria that cannot be routinely cultured in blood with current techniques [2].

The term 'mycotic' aneurysm is actually a misnomer; a more accurate term is endovascular infection or infective vasculitis. The term 'mycotic' first originated in 1885, when Sir William Osler, in his gulstonian lectures, described a patient with valve vegetations and 
Clinical Profile and the Management of Mycotic Superior Mesenteric Artery (SMA) Aneurysm in Infective Endocarditis: A Potential Tsunami

four aortic aneurysms whose morphology had a fungal resemblance. The most commonly affected organs due to embolism secondary to IE include the central nervous system (48-65\%), extremities (30\%), spleen (19-32\%), and kidney (6-14\%). Less commonly affected structures or organs include the lungs (14\%), coronary vessels (6\%), liver (3-11\%), bone and joints $(11 \%)$, iliac arteries $(6 \%)$ and mesenteric arteries $(3 \%)[3,4]$.

Visceral artery aneurysms as a result of arterial degenerative diseases are rare and carry an incidence of $0.1 \%-2 \%$ in medical literature [5]. They are mostly asymptomatic and detected incidentally during diagnostic imaging. SMA and IMA aneurysms are symptomatic and often present acutely [6]. Pentad of abdominal pain, pyrexia of unknown origin, malaise, weight loss, and nausea remains the most convincing presentation of mycotic aneurysms. The mortality rate of SMAAs is $\sim 30 \%$ and the rate of rupture is $\sim 50 \%$ with a resultant mortality rate of $60 \%$ [7].

Surgical replacement for native valve endocarditis is indicated in patient with severe valvular dysfunction, thromboembolism or sepsis. These patients benefit from early surgical intervention [2]. The type of prosthetic valve to be used in infective endocarditis (mechanical or bioprosthesis) is controversial. Consensus guidelines for prosthesis selection in infective endocarditis recommend bioprosthetic or mechanical valve replacement based on life expectancy and co-morbidities. The possibility of recurrence of IE in replaced valves is a concern. Recurrence of IE has been documented to be $\sim 10 \%$ over 10 yrs period. Various studies have shown no difference between recurrences of IE in bioprosthesis and mechanical prosthesis $[4,8]$.

Indications for treatment of visceral artery aneurysm (VAA) include symptomatic VAA and asymptomatic VAA greater than $2 \mathrm{~cm}$, rapid expansion (> $0.5 \mathrm{~cm} /$ week), and pregnancy, women of childbearing age or liver transplantation. The first surgical treatment of VAA aneurysm was reported by De Bakery and Cooley in 1953 [9]. Ligation of the vessels that enter and exit the aneurysm is one of the more common surgical approaches to treat SMA aneurysms [10]. Collaterals from celiac artery and inferior mesenteric artery (IMA) are often adequate to prevent intestinal ischemia in proximal aneurysms. However, in distal segment aneurysms, as in this case, collaterals were inadequate, leading to bowel ischaemia.

The endovascular approach is another modality that has gained attention in recent years, but its use in mycotic SMAA is limited. Apart from anatomical and technical challenges, insertion of a foreign body into a hostile (infected) tissue can aid in further bacterial colonization leading to relapse of infection [11]. Currently, endovascular procedures are indicated only in high-risk individuals who cannot tolerate general anaesthesia or open surgery [12]. Aneurysms can also be treated using embolization coils, ethylene-vinyl alcohol copolymer (EVOH) stents, multilayer stents, stent grafts and histoacryl glue or a combination of these methods [13-15].

\section{CONClusion}

Mycotic aneurysm of superior mesenteric artery is a rare complication of infective endocarditis. Patient may have a varied presentation. Early diagnosis and timely surgical intervention is the most effective treatment to prevent serious complications such as aneurysmal rupture and death.

\section{ACKNOWLEDGEMENT}

The authors are thankful to the patient for consenting us to publish this report. We also thankful to the staff of Clinical and Interventional Cardiology Department, Lilavati Hospital and Research Centre for their support during the study.

\section{REFERENCES}

[1] Pulli W, Dorigo N, Troisi N, Pratesi AA. (2008).Surgical treatment of visceral artery aneurysm: a 25-year experience. J Vasc Surg. 48(2):334-42.

[2] Skalweit MJ (2016).Culture Negative Endocarditis: Advances in Diagnosis and Treatment, Contemporary Challenges in Endocarditis. Available from: https://www .inte chopen. Com/books/contemporary-challengesin-endocarditis/culture-negative- endocarditisadvances-in-diagnosis-and-treatment. DOI: 10.5772/64920.

[3] Piasek E, Sojka M, Kuczyńska M, Światłowski Ł, Drelich-Zbroja A, et al (2018).Visceral artery aneurysms-classification, diagnosis and treatment. J Ultrason. 18 (73): 148-151.

[4] de Troia A, Mottini F, Biasi L, Azzarone M, Tecchio T, et al (2016). Superior Mesenteric Artery Aneurysm Caused by Aortic Valve Endocarditis: The Case Report and Review of 
Clinical Profile and the Management of Mycotic Superior Mesenteric Artery (SMA) Aneurysm in Infective Endocarditis: A Potential Tsunami

the Literature. Vasc Endovascular Surg. 50 (2):88-93.

[5] Grotemeyer D, Duran EJ, Park N, Hoffmann D, Blondin F,Iskandar F, et al (2009). Visceral artery aneurysms follow-up of 23 patients with 31 aneurysms after surgical or interventional therapy. Langenbecks Arch Surg. 394(6):10932000.

[6] Toyoda N, Itagaki S, Tannous H, Egorova NN, Chikwe J (2018). Bioprosthetic Versus Mechanical Valve Replacement for Infective Endocarditis: Focus on Recurrence Rates.The Annals of Thoracic Surgery. 106 (1):99-106.

[7] Drescher R, Köster O, von Rothenburg $T$ (2006). Superior mesenteric artery aneurysm stent graft. Abdom Imaging. 31(1):113-116.

[8] Zouizra Z, Biaou G, El Mardouli M, El Haouati R, Boumzebra D (2018). A real dilemma: Management of multiple mycotic visceral aneurysms revealing severe endocarditis. Ind $\mathbf{J}$ Vasc Endovasc Surg. 5(1):50-52.

[9] De Bakey ME, Cooley DA (1953). Successful resection of mycotic aneurysm of superior mesenteric artery; case report and review of literature. Am Surg. 19:202-12.

[10] Marone EM, Mascia D, Kahlberg A, Brioschi C, Tshomba Y, Chiesa R (2011). Is open repair still the gold standard in visceral artery aneurysm management? Ann Vasc Surg. 25 (7):936-946.
[11] Fisk M, Peck LF, Miyagi MJ, Steward MJ, Lee SF, Macrae MB, et al (2012). Mycotic aneurysms: a case report, clinical review and novel imaging strategy. QJM 105(2):181-188.

[12] Branchi V, Meyer C, Verrel F, Kania A, Bölke E, and Semaan A, et al (2019). Visceral artery aneurysms: evolving interdisciplinary management and future role of the abdominal surgeon. Eur J Med Res. 24: 17.

[13] Bratby MJ, Lehmann ED, Bottomley J, et al (2006). Endovascular embolization of visceral artery aneurysms with ethylene-vinyl alcohol (Onyx): a case series. Cardiovasc Intervent Radiol. 29 (6):1125-1128.

[14] Künzle S, Glenck M, Puippe G, Schadde E, Mayer D, Pfammatter T (2013). Stent graft repairs of visceral and renal artery aneuryms are effective and result in long term patency. J Vasc Interv Radiol. 24 (7):989-996.

[15] Bracaie UM, Narese D, Ficarelli I, et al (2017). Stent-assisted detachable coil embolization of wide-necked renal artery aneurysms. Diagn Interv Radiol. 23 (1):77-80.

Citation: Prakash Sanzgiri, et al, Clinical Profile and the Management of Mycotic Superior Mesenteric Artery (SMA) Aneurysm in Infective Endocarditis: A Potential Tsunami. ARC Journal of Cardiology. 2019; 5(2): 23-27. doi:dx.doi.org/ 10.20431/2455-5991.0502005.

Copyright: (c) 2019 Authors. This is an open-access article distributed under the terms of the Creative Commons Attribution License, which permits unrestricted use, distribution, and reproduction in any medium, provided the original author and source are credited. 